\title{
Modern techniques of measure and control of deformations - an experimental test: Senerchia landslide
}

\author{
M. Caprioli \& G. Strisciuglio \\ Dipartimento di Vie e Trasporti, Politechnic of Bari, Italy
}

\begin{abstract}
Control of movements and deformations is based on the comparison between surveys repeated in different times and in particular on the determination of a certain number of points. The topographical method represents one of the possible solutions in order to reach such an aim. A control network is constituted by reference points (located in stable zones) and control points (located in unstable zones). The movements can be of two kinds: "relative" between the control points (network deformations) and "absolute" (control points respective to the reference network). Its necessary, therefore, to estimate, during the time, the stability of reference points. In this work the stability of reference network located in Senerchia (Southern Italy) and constituted for the monitoring of a landslide area with static GPS - has been investigated. Both the classical and the Bayesian statistical analysis have been executed on the network in order to identify small movements in comparison with accuracy of the measurements. The application of the Bayes theory on the network has confirmed its stability.

Keywords: deformation analysis, statistical inference, Bayesian approach, GPS.
\end{abstract}

\section{Network in deformation analysis}

Control of movements and deformations is based on the comparison between surveys repeated in different times and particularly on the determination of the variation between the position of the points at the beginning and at the end of the considered period. The topographical method represents one of the possible ways to reach this aim. It can synthetically be summarized in the following phases: choice of the technique and instrumentation to be used, planning of the network configuration, monumentation of the vertexes, execution of the measurements 
(manuals/automatic), data processing, deformation analysis using statistical inference, optimization of geodetic network. Nowadays the development of technology allows a great range of solutions for survey execution (digital levelling, motorized TPS, laser scanners, SAR, GPS with RTK transmission): our survey has been performed with GPS instrumentation. Static GPS is the most adopted method, especially when high precision is requested: the standard precision in relative positioning is $10^{-6} \mathrm{D}$ or $10^{-7} \mathrm{D}$ in some experiences, with particular elaborations and satellite configurations.

\begin{tabular}{|c|c|c|c|c|}
\hline METHOD & TIME & BASELINES & ACCURACY & $\begin{array}{c}\text { RATE } \\
{[\mathrm{s}]}\end{array}$ \\
\hline \multirow{4}{*}{ STATIC } & $30^{\prime}$ & $<10 \mathrm{Km}$ & \multirow{4}{*}{$10^{-6} \div 10^{-8}$} & \multirow{4}{*}{$15 \div 60$} \\
\hline & $1 \mathrm{~h}$ & $10 \mathrm{Km}$ & & \\
\hline & $3 \div 4 \mathrm{~h}$ & $20 \div 30 \mathrm{Km}$ & & \\
\hline & variabile & $>100 \mathrm{Km}$ & & \\
\hline \multirow{2}{*}{ FAST STATIC } & $20^{\prime} \div 30^{\prime}(\mathrm{L} 1)$ & \multirow{2}{*}{$10 \div 15 \mathrm{Km}$} & \multirow{2}{*}{$10^{-6}$} & \multirow[t]{2}{*}{$5 \div 15$} \\
\hline & $6^{\prime} \div 8^{\prime}$ & & & \\
\hline STOP\&GO & $<1^{\prime}$ & $<10 \mathrm{Km}$ & $\mathrm{cm}$ & $1 \div 5$ \\
\hline KINEMATIC & --- & $\begin{array}{c}<2 \mathrm{Km} \\
\text { (radio modem) }\end{array}$ & $\mathrm{cm}$ & $1 \div 5$ \\
\hline
\end{tabular}

Figure 1: GPS acquisition.

In deformation analysis, the optimal network configuration has a strategic role to reach desired accuracies, to define an efficient program of measurements, to establish a proper mathematical model for the description of investigated phenomena. A topographical network must be planned keeping in mind that it is necessary to effect measurements in hyper determined number, in order to reach evaluation of measurements uncertainty and of standard deviations. The redundant scheme of the network allows to make a preliminary analysis, with the aim to determine the presence of gross errors, and, in second phase, the network adjustment with the aim to get $3 \mathrm{D}$ points position and the estimated value of the accuracies with which they has been determined. In this paper the adjustment method is based on Gauss-Markov model. At the end of the adjustment process, it can be determined the interval of confidence for the respect of the position of every unknown point of the network (error ellipse/ellipsdoids). The analysis of the residuals is not enough to individualize gross errors: Date Snooping method (Baarda, [12]) proposes the use of normalized residuals of the measurements and local redundancy (contribution of every single observation to the general redundancy of the network). Control network is constituted by reference vertexes (points situated in stable zones) and by control vertexes (points situated in not stable zones, inside the monitoring zone). There are two typologies of movements: relative among the control points (deformations of the control network) and absolute (control points in comparison to a stable structure or to the reference frame. It's important, therefore, to appraise, during the time, the stability of the reference vertexes. In this paper, the aim is to verify the stability a control network, constituted for monitoring a landslide area. The investigation results interesting especially for the particular location of a point. 


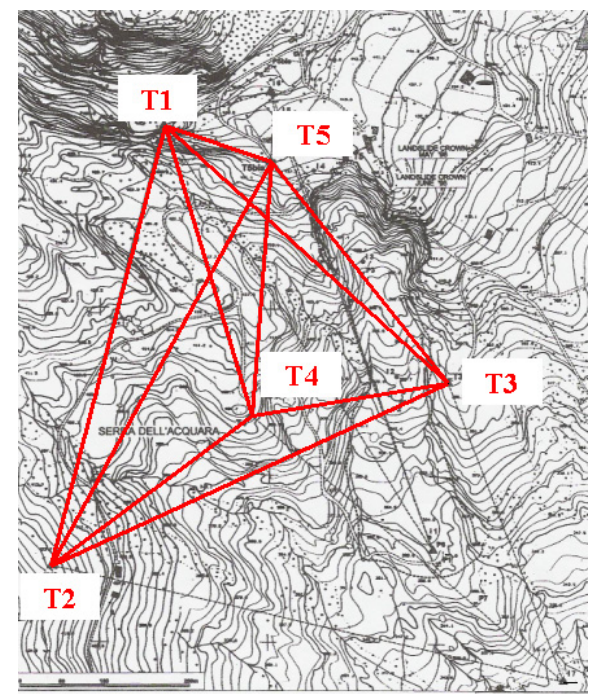

Figure 2: Control network of Senerchia.

\section{Experimental test}

Experimental test has been effected using campaigns of measurements related to the landslide of Serra dell' Acquara-Vadoncello, Senerchia (SW Italy).

The aim is to verify the application of the theoretical studies to a series of observations of control network, obtained using modern techniques of survey. The area of study is located in a zone of the southern Appennino, characterized by catastrophic events in 1980 and 1996, due to unstable nature of the area. The choice of such site is tied up to the presence of database coming from research works effected in the past years: data have been compared and integrated with those coming from the surveys directly effected. In this context, it is inserted the topographical study of the zone, finalized to the geodetic monitoring of small control network constituted by five vertexes.

The particular location of vertex T4 (outside the landslide area of 1993 but inside the 1980 surface) requests a closer examination during the analysis, justifying the chance to use innovative tools/theory in the analysis of the observations (Bayes theory). The objective is to verify the stability of all the vertexes retained fixed and used as stations of distantiometric measurements to control position of points belonging to the landslide mass. Reference vertexes have been materialized through the construction of specific $3 \mathrm{D}$ concrete monuments (circular section $30 \mathrm{~cm}$, height $120 \mathrm{~cm}$ ) for the stable positioning of GPS antenna. During survey campaigns the following instrumentation has been used:

- $\mathrm{n}^{\circ} 2$ LEICA GPS1200 (GX1230, 12 L1s + 12 L2s / WAAS / EGNOS) with Smart Track antenna AX 1202, Ground incorporated plane;

- $\mathrm{n}^{\circ} 1$ GPS LEICA System 500- SR530 double frequency (L1 codes C/A; L2 code P), antenna AT502, controller TR500; 


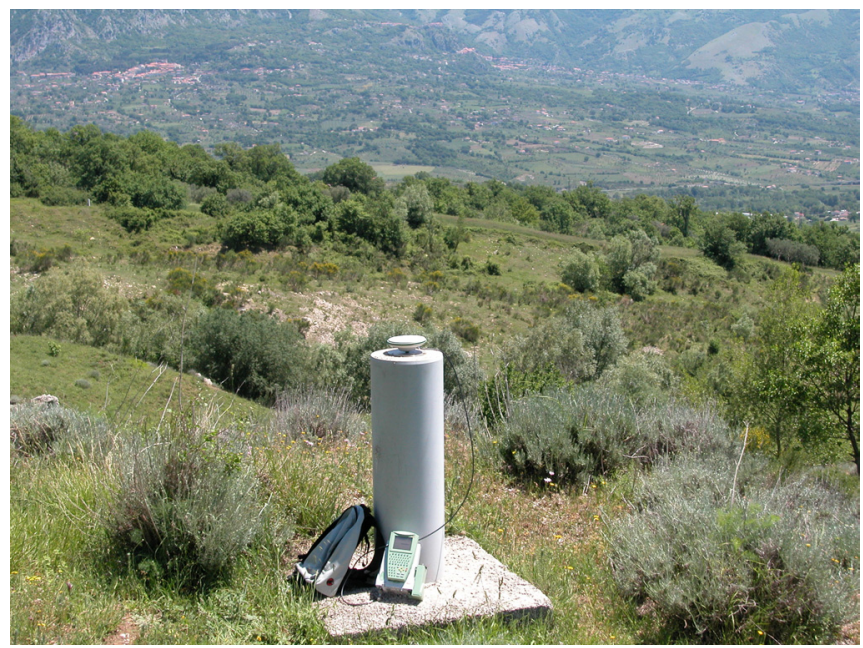

Figure 3: $\quad$ Vertex T4 of the network.

- n² GPS Trimble 4700 (L1 code C/A,) with controller TSC1;

- $\mathrm{n}^{\circ} 1$ Smartstation with Smart Antenna.

Use of Smartstation has allowed one to integrate GPS survey, primary source of data in the experimental test, with classical topographical data: angles and distances. Every GPS survey has been conducted in 3 independent static sessions, duration of 45 minutes. The tools rate has been planned of 10 seconds. The satellite configuration and the climatic conditions are optimal during the whole execution of survey operations. Download and post-processing phase have been executed in Geomatics Laboratory of Polytechnic of Bari, using software furnished with the receivers (Leica Geo Office 2.1 and Trimble Geomatics Office 1.01). All the data have subsequently imported in the second application to perform treatment according to the "single base" approach. At the end, the test of ratio has performed: it has broadly been always overcome the limit of 1.5 for GPS measurements. The network adjustment has been executed with least square method.

\section{Classical analysis of the network movements}

It has been decided to conduct on control points both the classical and the Bayesian analysis. While classical analysis has the purpose to quantify the entity of movements, the Bayesian approach has the purpose to identify the zones interested by significant movements. The simplification of the statistic problem is a necessary condition to allow the comparison among the results: therefore, the coordinates $(\mathrm{N}, \mathrm{E}, \mathrm{h})$ of every vertex $\mathrm{Pj}(\mathrm{j}=1, \ldots, 5)$ of the network have been considered separately. The first hypothesis consists of considering not correlated coordinates in space and time (among the different sessions of measurements). 


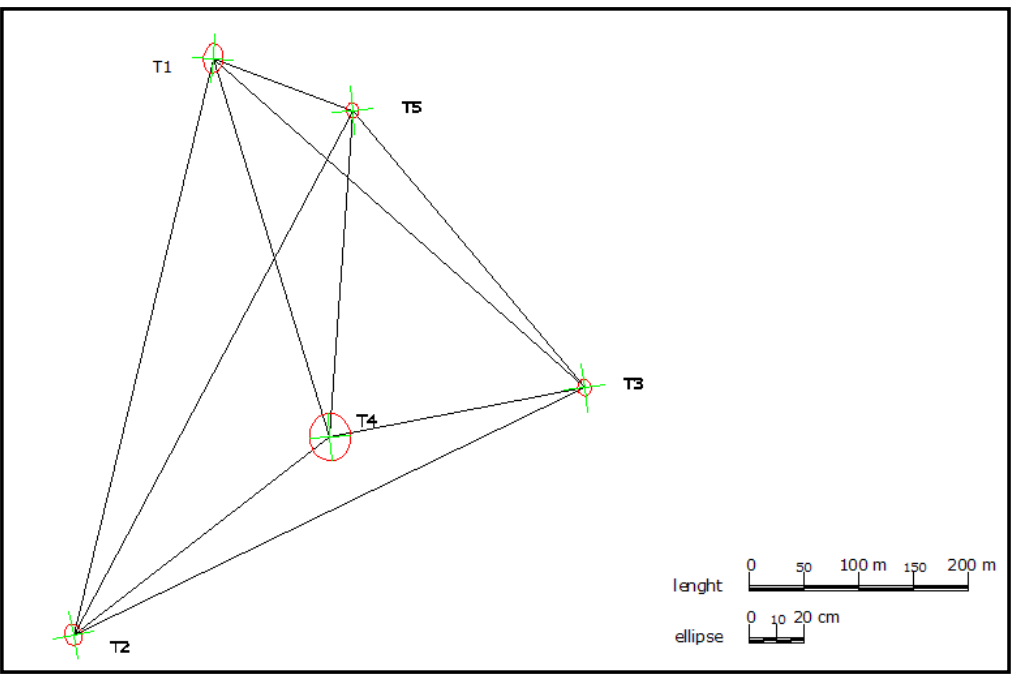

Figure 4: $\quad$ GPS network and error ellipses.

The hypothesis to be submitted to inference is that movements are not significant:

$$
H_{0}: \delta x=0
$$

It is possible to build the following statistic parameter:

$$
Z \sim \frac{\Delta x}{\sqrt{\sigma_{v 1}^{2}+\sigma_{v 2}^{2}}}
$$

Fixed the level of $\alpha=5 \%$ (corresponding to $Z=1.96$ ), the hypothesis $H_{0}$ is approved (so the movement of the point is not significant) if:

$$
-Z_{\alpha / 2} \cdot \sqrt{\sigma_{v 1}^{2}+\sigma_{v 2}^{2}} \leq \Delta x \leq Z_{\alpha / 2} \cdot \sqrt{\sigma_{v 1}^{2}+\sigma_{v 2}^{2}} .
$$

The calculation has been effected considering all the sessions of measurements that are divided in: GPS data coming from the effected surveys (fifth and sixth session); GPS data coming from a series of campaigns of measurements (first 4 sessions) conducted starting from the 1996.

Sessions of measurements are totally 6: all data are the result of network adjustment effected with least square method. 


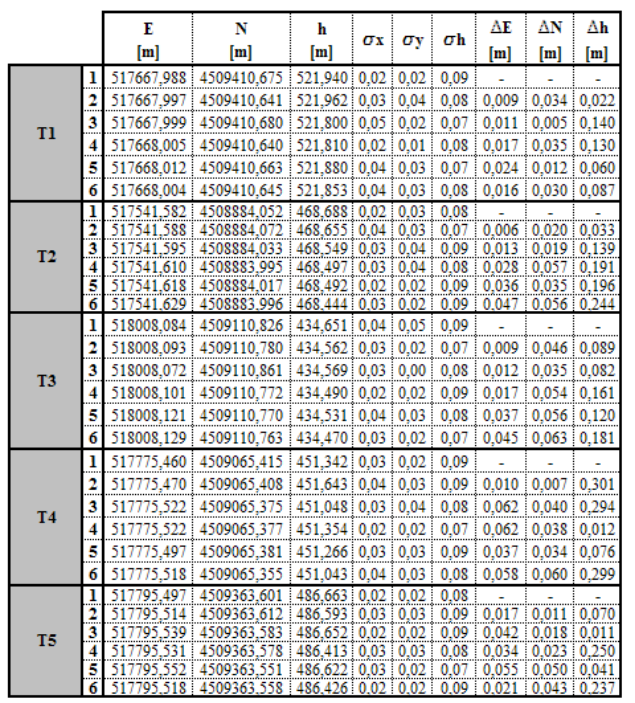

Figure 5: $\quad$ GPS post-processed data.

In the following charts for every point of the network and for every coordinate $(\mathrm{E}, \mathrm{N}, \mathrm{h})$, the observed values are the following:

$$
\begin{aligned}
& Z_{o s s}=\frac{\Delta E}{\sqrt{\sigma_{v 1}^{2}+\sigma_{v i}^{2}}} \\
& Z_{\text {oss }}=\frac{\Delta N}{\sqrt{\sigma_{v 1}^{2}+\sigma_{v i}^{2}}} \\
& Z_{o s s}=\frac{\Delta h}{\sqrt{\sigma_{v 1}^{2}+\sigma_{v i}^{2}}}
\end{aligned}
$$

As concerns Figure 6, on celestial background there are values of $Z_{\text {oss }}$ that underline the presence of a possible significant movement in comparison to the first campaign of measurements.

Classical analysis furnishes unequivocal results on the planimetric coordinates, because the values of $Z_{\text {oss }}$ are inferior to the threshold value (1.96) denoting absence of movement. Some problem arises on the height, as it was possible to expect for GPS observations characteristics.

\section{Bayesian analysis for the movements of the network}

In Bayesian analysis, the only coordinate $h$ got by the network adjustment in different times has been considered: classical analysis is not able to clearly 
define the vertical behavior of control vertexes. The quantities to be considered therefore, are movement $\Delta \mathrm{h}$ among the different sessions of all the not-fixed points:

$$
(\Delta h)_{P_{J}}=\left(h_{i}-h_{1}\right)_{P_{j}}
$$

with $\mathrm{i}=2,3,4,5,6$ and $\mathrm{j}=1,2,3,4,5$.

$\mathbf{E}$

\begin{tabular}{|c|c|c|c|c|c|}
\hline \multicolumn{1}{c|}{} & $\mathbf{2}>\mathbf{1}$ & $\mathbf{3}>\mathbf{1}$ & $\mathbf{4}>\mathbf{1}$ & $\mathbf{5}>\mathbf{1}$ & $\mathbf{6 > 1}$ \\
\hline T1 & 0,25 & 0,20 & 0,61 & 0,54 & 0,36 \\
\hline T2 & 0,13 & 0,36 & 0,78 & 1,27 & 1,30 \\
\hline T3 & 0,18 & 0,24 & 0,38 & 0,65 & 0,90 \\
\hline T4 & 0,20 & 1,46 & 1,32 & 0,87 & 1,16 \\
\hline T5 & 0,46 & 1,47 & 0,93 & 1,52 & 0,73 \\
\hline
\end{tabular}

$\mathbf{N}$

\begin{tabular}{|c|c|c|c|c|c|}
\cline { 2 - 7 } \multicolumn{1}{c|}{} & $\mathbf{2 > 1}$ & $\mathbf{3 > 1}$ & $\mathbf{4 > 1}$ & $\mathbf{5 > 1}$ & $\mathbf{6 > 1}$ \\
\hline T1 & 0,76 & 0,18 & 1,57 & 0,33 & 0,83 \\
\hline T2 & 0,47 & 0,38 & 1,14 & 0,97 & 1,55 \\
\hline T3 & 0,85 & 0,70 & 1,00 & 0,96 & 1,17 \\
\hline T4 & 0,19 & 0,89 & 1,34 & 0,94 & 1,66 \\
\hline T5 & 0,31 & 0,64 & 0,64 & 1,77 & 1,52 \\
\hline
\end{tabular}

h

\begin{tabular}{|c|c|c|c|c|c|}
\hline & $\mathbf{2}>\mathbf{1}$ & $\mathbf{3}>\mathbf{1}$ & $\mathbf{4}>\mathbf{1}$ & $\mathbf{5}>\mathbf{l}$ & $\mathbf{6 > 1}$ \\
\hline T1 & 0,18 & 1,23 & 1,08 & 0,53 & 0,72 \\
\hline T2 & 0,31 & 1,15 & 1,69 & 1,63 & 2,03 \\
\hline T3 & 0,78 & 0,68 & 1,26 & 1,00 & 1,59 \\
\hline T4 & 2,36 & 2,44 & 0,11 & 0,59 & 2,48 \\
\hline T5 & 0,58 & 0,09 & 2,21 & 0,39 & 1,97 \\
\hline
\end{tabular}

Figure 6: $\quad$ ZOSS reported to the $\mathrm{E}, \mathrm{N}$ and $\mathrm{h}$ coordinates.

We can suppose that $\Delta \mathrm{h}$ follow a normal distribution:

$$
\Delta h \sim N\left(\delta h, \sigma_{h}^{2}\right)
$$

Parameters of this distribution are average (unknown) and the known variance factor that is obtained by the effected least square adjustment. For every point of the network we have:

$$
\Delta h=h_{i}-h_{1}=\delta h+\sigma_{h}
$$

$\delta h$ is an aleatory variable, distributed according to a normal density of probability, of average $\mu$ and variance $\sigma_{0}^{2}$ :

$$
\delta h \sim N\left(\mu, \sigma_{0}^{2}\right)
$$

Parameters of this distribution (prior function of Bayesian theory), are information of the problem (they will have been fixed during the numerical 
analysis). Since we effect an analysis of vertical movements of points in landslide area, it is supposed that the movements are void or downward. Then, considering an axle opportunely directed, it is reasonable to impose previously a further condition: $\delta h \geq 0$.

This condition becomes complicated to impose in classical inference. Bayesian theory, explicited all the terms, is:

$$
\begin{gathered}
f(\delta h / \Delta h)=\frac{f(\Delta h / \delta h) \cdot f(\delta h)}{K}= \\
=\frac{1}{A+B} \cdot\left\{\frac{P_{0}}{\sqrt{2 \pi} \cdot \sigma_{h}} \cdot e^{-\frac{\Delta h^{2}}{2 \sigma_{h}^{2}}} \cdot \delta(\delta h)+\frac{\vartheta(\delta h)}{\sqrt{2 \pi} \sigma_{h} \sigma_{0}} \cdot e^{-\frac{\left(q_{h} \Delta h^{2}+q_{0} \mu^{2}-\bar{q} \cdot m^{2}\right)}{2}} \cdot \frac{e^{-\frac{(\delta h-m)^{2}}{2 \overline{\sigma^{2}}}}}{\sqrt{2 \pi} \cdot \bar{\sigma}} \cdot \bar{\sigma}\right\} .
\end{gathered}
$$

The analysis of the movements through the Bayesian approach can reduce then to the comparison between the two quantities:

$$
P(\delta h=0 \backslash \Delta h)=\frac{A}{A+B} \quad \text { and } \quad P(\delta h>0 \backslash \Delta h)=\frac{B}{A+B} .
$$

From the comparison between the two quantities above, it is to be verified which of the two alternatives has greater probabilities to verify:

$$
A \equiv \frac{P_{0}}{\sqrt{2 \pi} \cdot \sigma_{h}} e^{-\Delta h^{2} / 2 \sigma_{h}^{2}} \text { and } B \equiv \frac{\bar{\sigma} \cdot e^{-\frac{\left(q_{h} \Delta h^{2}+q_{0} \mu^{2}-\bar{q} m^{2}\right)}{2}}}{\sqrt{2 \pi} \sigma_{h} \sigma_{0}} \cdot\left[1-\operatorname{erf}\left(-\frac{m}{\bar{\sigma}}\right)\right] \text {. }
$$

All the terms that appear in these expressions are numerically determinable; particularly, the known values of $\mu$ and of $\sigma_{0}$.

\begin{tabular}{|c|c|}
\hline $\mathrm{P}(\mathrm{dh}>0)$ & MOVEMENT \\
\hline$<0.475$ & no \\
\hline$>0.525$ & yes \\
\hline $0.475 \div 0.525$ & doubt \\
\hline
\end{tabular}

Figure 7: Bayesian analysis.

In classical analysis the statistic test has been performed considering a level of significance $\alpha=5 \%$. The interpretation of the results in the Bayesian analysis happens as shown in Figure 7.

Six different elaborations have been effected, according to the initial hypotheses, always considering the only $h$ coordinate, using a simplified model 
and selecting different values of prior data. They calculated the values of $\mathrm{P}(\mathrm{dh}>0)$ consequential from the comparison of all the measurement sessions with the first assumed as reference.

Later, we present the results deriving from the sixth elaboration (Figure 8), in which we consider the following data prior: $\sigma_{0}=0.05 \mathrm{~m}$ for every network vertex, $\mu$ equal to the average of the $\Delta \mathrm{h}$ in correspondence of every vertex .

\begin{tabular}{|c|c:c:c:c:c|}
\hline & $\mathbf{2 > 1}$ & $\mathbf{3 > 1}$ & $\mathbf{4 > 1}$ & $\mathbf{5 > 1}$ & $\mathbf{6 > 1}$ \\
\hline T1 & 0,214 & 0,000 & 0,001 & 0,034 & 0,012 \\
\hdashline $\mathbf{T} 2$ & 0,041 & 0,000 & 0,000 & 0,000 & 0,001 \\
\hdashline $\mathbf{T} 3$ & 0,002 & 0,006 & 0,000 & 0,000 & 0,000 \\
\hdashline T4 & 0,007 & 0,022 & 0,100 & 0,003 & 0,030 \\
\hdashline T5 & 0,016 & 0,295 & 0,005 & 0,040 & 0,001 \\
\hline
\end{tabular}

Figure 8: Bayesian analysis.

Prior data has been select exploiting the accumulated experience and knowledge of the phenomenon coming from a first analysis of raw data. It immediately emerges that, in all the elaborations, the numerical value of $\mathrm{P}(\mathrm{dh}>0)$ calculated for network vertexes underlines that the relative movement is "not significant". The doubts appeared after the application of classical analysis has been resolved: there is no vertical movement of the vertexes.

\section{Conclusions}

The application of Bayes theory on the network has confirmed its stability during the time: significant movements are not present. The vertexes are stable and therefore usable as stations of measurements to control points position in landslide area. The application is limited only to the vertical direction because of classical analysis has already clarified the absence of planimetric movements. Results coming from Bayesian analysis are characterized by great coherence: also varying some prior data they appear stable. It is clarified, with Bayesian analysis, the behaviour of T4 vertex but it appears a necessary constant monitoring on it, due to its particular positioning. The application of Bayes theory is efficient for the aim of this paper: information insertion (critical phase of the procedure) has allowed to conduct a statistical analysis finalized to detect small movements in comparison to the standard deviation of the residuals.

This method must not be considered as substitutive of classical analysis but integrative, because it uses the values of the parameters got with least square method. The difficulty of the Bayesian approach is to fit probability distribution to data and to make inferences from parameter distributions. 


\section{References}

[1] Augutis, J., Zutautaite, I., Alzbutas, R., Uspuras, E., Iterative estimation of reliability parameters using the Bayesian approach, Risk Analysis IV, WIT Press 2004, ISBN 1-85312-736-1.

[2] Barbarella, M. \& Radicioni, F., Kalman Filtering in levelling: an application, Global and Regional Geodynamics, Springer-Verlag, NY, 1990.

[3] Barbarella, M., Canuti, P., Fiani, M., \& Vannocci, P., Controllo di movimenti gravitativi profondi, XIII GNGTS Proceedings, Rome, 551562 .

[4] Bernstein, S., \& Bernstein, R., Statistica Inferenziale, McGraw-Hill, 2003.

[5] Caprioli, M., Importance and role of the GPS permanent national network for topographic and professional application, ASI-CGS, Matera, 1997.

[6] Caprioli, M., Stability controls and deformations measurements in civil engineering works, Allied Publishers Ltd, ISBN 81-7764-245-6.

[7] Cina, A., GPS - Principi, modalità e tecniche di posizionamento, Celid, ISBN 88-7661-417-6, 2000.

[8] Erol, S., Erol, B., Ayan, T., A general review of the deformation monitoring techniques and a case study: analysing deformations using GPS/levelling, ISPRS-WG VII/5,XXth ISPRS Congress Geo-Imagery Bridging Continents, Istanbul, Turkey, 12-23 July 2004.

[9] Pinto, L., \& al., Controllo delle deformazioni: un approccio statistico di tipo Bayesiano, VII ASITA National Conference, 2003.

[10] Sacerdote, F., Albertella, A., Cazzaniga, N., Applicazione di metodi bayesiani per il controllo delle deformazioni, VIII ASITA National Conference, 2004.

[11] Trizzino, R. \& Sorgente, M., Ottimizzazione di una rete di controllo GPS per il monitoraggio di una frana, Proceedings of International Conference "Prevention of Hydrogeological Hazards: the Role of Scientific Research", 1996.

[12] Baarda, W., A testing procedure for use in the geodetic network, Neth. Geod. Comm., n5, 1968. 